\title{
Three Year Study of Infection Profile and Antimicrobial Resistance Pattern from Burn Patients in Southwest Iran
}

This article was published in the following Dove Press journal:

Infection and Drug Resistance

\author{
Amir Emami (D) \\ Neda Pirbonyeh (1D \\ Abdolkhalegh Keshavarzi $\left(\mathbb{D}^{2}\right.$ \\ Fatemeh Javanmardi (D) \\ Sedigheh Moradi Ghermezi' \\ Tayyeb Ghadimi (iD ${ }^{3}$ \\ 'Microbiology Department, Burn and \\ Wound Healing Research Center, Shiraz \\ University of Medical Sciences, Shiraz, \\ Iran; ${ }^{2}$ Surgery Department, Burn and \\ Wound Healing Research Center, Shiraz \\ University of Medical Sciences, Shiraz, \\ Iran; ${ }^{3}$ Burn Research Center, Surgery \\ Department, Iran University of Medical \\ Sciences, Tehran, Iran
}

Introduction: Burn wound infections, as one of the most important risk factors, cause serious complications in burns. Hence, the focus of medical care should be preventing infections and resistant isolates. The current study investigates the prevalence of infectious agents and antimicrobial resistance patterns during three years.

Materials and Methods: A total of 960 isolates were collected from different sample kinds cultured for 615 burn patients who were hospitalized during January 2016 to December 2018 in Amir-Al-Momenin Burn Center. The type of microorganism and their antibiotic resistance patterns were identified by microbiological tests and the standard disk-diffusion method according to the introduced standard techniques.

Results: Incidence of positive growth was seen more in males than in females. Most of the burns encountered were due to flame injuries (35.4\%). Based on the diversity of bacterial isolates, Pseudomonas aeruginosa was the most frequent pathogen (49.9\%), followed by Klebsiella sp. (9.7\%), Acinetobacter sp. (7.2\%) and Staphylococcus aureus (6.5\%). The trend of resistance of meropenem was declining in P. aeruginosa isolates. Klebsiella sp. as the second most prevalent agent showed a high level of resistance to the studied antibiotics. The antibiogram results for $S$. aureus isolates showed an increasing trend in MRSA isolates. Conclusion: By evaluating the infectious agent, it was found that although frequencies of microorganisms and resistant isolates were a little high, performing a multidisciplinary approach controls the trend during the study period. These achievements have been gained due to a strict politicized infection control and stewardship program in the appointed burn center.

Keywords: nosocomial infections, antibiotic resistance pattern, burn

\section{Introduction}

Nosocomial infections are one of the most serious complications in hospitalized patients contributing to mortality and morbidity rates. Burn patients, due to the features of their injuries, are exposed to hospital-associated infections more than other hospitalized patients. ${ }^{1}$ Losing the protective skin barrier as the first line of defense and defects appearing in the patient's immune system make the injured tissue a proper environment for microbial growth. It has been illustrated that about $30-80 \%$ of burn patients are threatened with nosocomial infections. ${ }^{2}$ Despite development in burn care over the last decades, infections remain the leading cause of death in this group of victims. According to previous reports, $42-65 \%$ of burn deaths are attributed to infections. ${ }^{3}$ Microorganisms associated with nosocomial infections have endogenous
Correspondence: Amir Emami Microbiology Department, Burn and Wound Healing Research Center, Shiraz University of Medical Sciences, Shiraz, Iran

Email emami.microbia@gmail.com 
or exogenous sources which recently are more related to health care staff and hospital setting environments. According to the different reports, the distribution trend of microorganisms in hospitalized patients is mostly changed during this time and almost changes from Gram-positive to Gram-negative bacteria and in long-term hospitalization leads to fungal infections. ${ }^{4}$ Literature reviews in burn patients have shown that the most common pathogens are Pseudomonas aeruginosa, Acinetobacter baumannii, Staphylococcus aureus and Klebsiellasp. Infections in burn patients should be considered of utmost importance since the following complications threaten their life: multiple organ dysfunction, sepsis, bacteremia and delay in the healing process of their injuries. In order to prevent infection, different strategies through an infection control program should be used and paid attention to. ${ }^{5}$

One of the biggest challenges and specific concerns about bacterial infections is their antimicrobial resistance pattern. The resistance microorganisms are able to combat antimicrobial agents, which causes ineffective treatment and perseverant spreading of infections. ${ }^{6,7}$ Based on different reports, it has been estimated that more than $70 \%$ of health care-associated infections are resistant to at least one of the first-line or common antimicrobial drugs used in hospital. ${ }^{8-10}$ Recently, multidrug resistance (MDR) is increasing in hospital settings, which is more associated with the following: 1) irregular and inappropriate use of antibiotics; 2) empirical start of antibiotic use without relation to infection sources; 3) prolonged use of antibiotics in a center without knowing the resistance pattern; 4) antibiotic therapy of infections without knowing the infectious agent and the antibiogram sensitivity pattern; and 5) relying on antibiotic therapy instead of clean care. Attention to the mentioned points and observing the principles of infection control and antibiotic stewardship will be helpful in decreasing the rate of morbidity and mortality and the burden of social and financial costs. ${ }^{11}$ As mentioned in the previous section, there are different reasons for the emergence of nosocomial multidrug resistance but the most important one is the use of broad-spectrum antibiotics blind empirically. Nowadays, ordering antibiotics especially thirdgeneration cephalosporins and some other antibiotics such as glycopeptides and polymyxins (the last resort of drug medication in resistance control) out of the stewardship programs will cause treatment failure. According to the antimicrobial resistance studies in Iran, although the resistance rate in hospital-acquired infections is not very high compared with other developing countries, but the same as global statistics, MDR isolates are increasing in our clinical centers, and infection control programs with comprehensive antibiotic stewardship plans must be performed seriously. ${ }^{12,13}$ To perform guidelines comprehensively, determining the pattern of infections and their resistance is a considerable issue in the aspect of epidemiological and clinical purposes and would be helpful for clean care and infection control in alternative use of antibiotics. In accordance with implementing the above policy, the recent study was conducted to determine the prevalence of nosocomial infections and related drug resistance pattern during three years in the major burn center of southwest Iran, Amir-Al-Momenin burn and wound healing hospital.

\section{Materials and Methods}

This cross-sectional study was conducted in burn patients hospitalized in Amir-Al-Momenin burn hospital affiliated with Shiraz University of Medical Sciences, Shiraz, Iran (SUMS). Over a period of three years from January 2016 to December 2018, patients of both genders, burn degree 2 and more, from every ward with no limited age were included in this study. Patients were excluded with any history of cancer, any immunocompromised diseases and using antibiotic drugs at the time of admission. A total of 3420 samples from 615 hospitalized patients with the following criteria were taken from hospitalized patients. Characteristics of patients are provided in Table 1. To evaluate the prevalence of nosocomial infection in the hospital wards, specific samples such as wound, urinary tract, sputum, nose, catheters and stool were taken from patients according to their conditions at least after three days of hospitalization. In order to evaluate better, more than one sample of different kinds was taken from some patients. All of the samples were examined for infections by standard and specific microbiological tests. Finally, a total of 960 samples were growth positive and included in the analysis. The distribution of bacteria in different wards during the study period is shown in Table 2 .

All growth bacteria were classified into Gram-negative /positive and identified based on Standard Microbiological procedures. According to the Clinical and Laboratory Standard Institute (CLSI 2018) guideline, antibiotic susceptibility tests were performed for all of the confirmed isolates by the disk-diffusion method. For this purpose, pure confirmed isolates were prepared in $0.5 \mathrm{McF}$ arland standard suspension. Each prepared isolate was then plated onto Muller-Hinton agar (Difcos) plates. Antibiotic disks (Oxoid) were applied to each plate based on the same protocol which has been listed in Table 3. According to the CLSI 
Table I Demographic Characteristics of Positive Growth Patients

\begin{tabular}{|l|l|l|l|l|l|}
\hline & Variables & $\mathbf{2 0 I 6 ( n = 2 I I )}$ & $\mathbf{2 0 I 7}(\mathbf{n = 2 4 6})$ & $\mathbf{2 0 I 8}(\mathbf{n = 1 5 8 )}$ & Total \\
\hline Demographics & Male/female & $130(61.6 \%) / 8 I(38.4 \%)$ & $155(63 \%) / 91(37 \%)$ & $88(55.7 \%) / 70(44.3 \%)$ & $373(60.7 \%) / 242(36.9 \%)$ \\
& Age & $29.44 \pm 22.03$ & $26.33 \pm 21.96$ & $31.82 \pm 19.56$ & $28.79 \pm 21.48$ \\
& TBSA & $31.46 \pm 20.85$ & $29.78 \pm 22.13$ & $38.89 \pm 22.55$ & $32.53 \pm 22.05$ \\
\hline \multirow{2}{*}{ Burn cause } & Flame & $76(40 \%)$ & $84(36.5 \%)$ & $58(54.2 \%)$ & $218(41.4 \%)$ \\
& Electrical & $5(2.6 \%)$ & $9(3.9 \%)$ & $8(7.5 \%)$ & $22(4.2 \%)$ \\
& Scald & $48(25.3 \%)$ & $69(30 \%)$ & $18(16.8 \%)$ & $135(25.6 \%)$ \\
& Chemical & $1(0.5 \%)$ & $2(0.9 \%)$ & $1(0.9 \%)$ & $4(0.8 \%)$ \\
& Hot object & $2(1.1 \%)$ & $4(1.7 \%)$ & $2(1.9 \%)$ & $8(1.5 \%)$ \\
& Explosion & $58(30.5 \%)$ & $62(27 \%)$ & $20(18.7 \%)$ & $140(26.6 \%)$ \\
\hline
\end{tabular}

Abbreviation: TBSA, total burn surface area.

guideline, methicillin-resistant $S$. aureus isolates can be determined by oxacillin. Oxacillin-resistant Staphylococci are resistant to all currently available $\beta$-lactam antimicrobial agents, with the exception of the newer cephalosporins with anti-MRSA activity, so testing of other $\beta$-lactam agents, except those with anti-MRSA activity, is not advised. ${ }^{14}$

Based on the guideline, for sensitive control, reference strains ATCC 25923 (S. aureus), ATCC 35218 (Escherichia coli), ATCC 27853 (P. aeruginosa) and ATCC 43816 (Klebsiella pneumonia) were used. These strains were obtained from the Department of Microbiology, Burn and Wound Healing Research Center, SUMS. The process and procedures of the study were approved by the Ethical Committee code: IR.SUMS.REC.1398.418.

\section{Statistical Analysis}

The numerical data were expressed with mean \pm standard deviation, while categorical data were indicated with frequency and percentage. All statistical analysis was conducted with SPSS 16 (SPSS Inc., Chicago, IL, USA).

\section{Results}

During the study period, according to the inclusion criteria of the study, a total of 960 (28\%) positive growth samples were collected from 615 burn individuals. Age ranges of included patients were estimated as 1-89 years with mean $28.79 \pm 21.48$ years. The average of percent total body surface area (TBSA\%) was 32.53 \pm 22.05 (range: 1-100). There were $373(60.7 \%)$ male and 242 (39.3\%) female patients. Most of the burns encountered were due to flame injuries $(218,35.4 \%)$. More details about the demographic information of included patients are provided in Table 1.

As mentioned before, isolates were collected from different specimens from different kinds and wards.
According to the data analysis of total data, it has been deducted that wound infection was in the highest (264/ $960,27.5 \%)$ incidence, followed by nasal (96/960, 10.0\%), tissue $(78 / 960,8.12 \%)$ and sputum (66/960, 6.87\%) respectively. Based on the diversity of bacterial isolates among the study period, $P$. aeruginosa was the most frequent and in the highest incidence $(49.9 \%)$, followed by Klebsiellasp. (9.7\%), Acinetobactersp. (7.2\%) and S. aureus $(6.5 \%)$.

A comparison of the trend of infectious agents in the time of study based on year and ward has been shown in detail in Table 2. According to the results, an increasing trend has been seen in $P$. aeruginosa isolates during the study time. This increase was mostly related to the ICU ward, while in the other wards this infection was almost controlled with no changes. Klebsiellasp. was the second nosocomial infection agent with no considerable changes in trend during the time of the study. The results for Acinetobactersp. as the third infectious agent showed that this infection has been controlled in recent years and has a reducing trend. $S$. aureus as the first common Grampositive infection in our burn center overall had a reducing trend in all studied wards. Moreover, the results of the analysis of samples showed that some other uncommon bacteria such as E. coli and Proteussp. are present which have been detected in samples other than wounds. Among the results, some other bacteria such as Enterobacteriaceae and haemolytic Streptococcusspp. have been detected in low prevalence $(<0.7 \%)$, which according to the variety of sample type have not been analyzed in detail. In order to detect new emergence infections, coagulase-negative Staphylococcusspp. (CoNS) were evaluated in more detail. A total of 65/213 (30.51\%) CoNS were found as Staphylococcus lugdunensis. This bacterium is already 


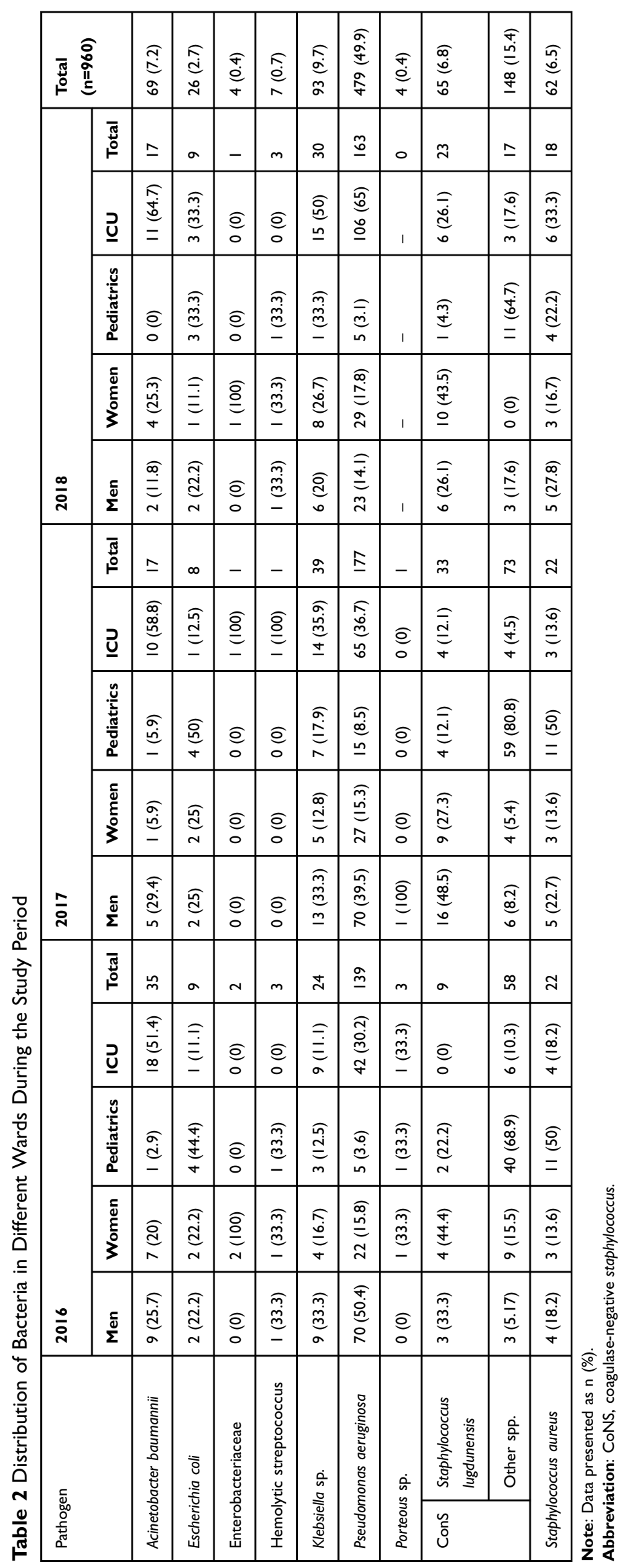




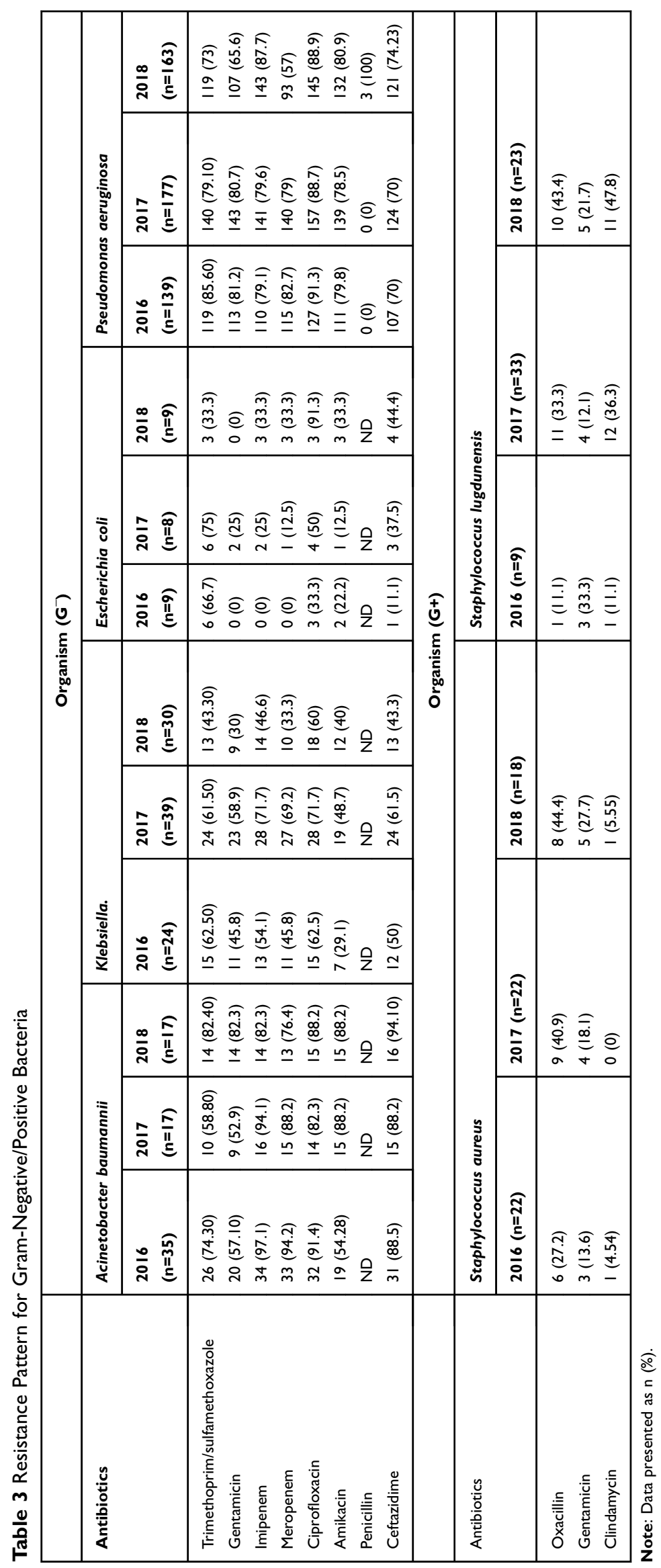


known as a cousin of $S$. aureus with the same pathogenicity potential.

The antibiogram sensitivity of the main nosocomial isolates was evaluated as the second aim of the study. Based on a common policy for each clinical setting, some drugs are in more common use for infection control. This is because the pattern of infection is almost known for each center and the infectious specialist almost used a defined empirical pattern for their patients. Based on this, the pattern of commonly used antibiotics which is listed in Table 3 was evaluated and analyzed. Based on this evaluation, carbapenem groups were detected as the choice drugs for $P$. aeruginosa isolates as the most prevalent pathogen. According to the analysis results for the resistance pattern of this bacterium to meropenem and imipenem, it has been deduced that the resistant trend is reducing for meropenem and there are no significant changes for imipenem among the years of study (2016-2018). For other antibiotic resistance patterns, detailed data are shown in Table 3.

According to the antibiogram resistance pattern for other prevalent Gram-negative bacteria, a high level of resistance in Klebsiella sp. was demonstrated especially in the second year of the study (2017), which seems to have been controlled and reduced in the next year.

For A. baumannii, total evaluations during the years of study have shown no significant changes in resistance pattern. The highest and lowest resistant rate was seen against imipenem (97.10\%) and amikacin (54.28\%) respectively during the years of study.

In the evaluation of Gram-positive isolates, Staphylococcus spp. were present in the highest rate. Based on the importance of different species of this genus, isolates were divided into three categories (S. aureus, S. lugdunensis and coagulase-negative Staphylococcus spp.) based on standard biochemical tests.

The antibiogram results for $S$. aureus isolates showed an increasing trend in MRSA isolates during the years of study based on the oxacillin test. In the following antibiotic sensitivity tests for MRSA isolates against vancomycin, it has been deduced that all were sensitive to vancomycin according to the MIC results. According to the pathogenic importance of S. lugdunensis species, antibiogram tests have been performed for this group of bacteria. All details related to this group of bacteria are shown in Table 3 .

\section{Discussion}

Infections are one of the most hazardous complications in all hospitalized patients, which are more pronounced in burn patients. Nowadays, despite improvement policies in treating burn patients, infections are still the main cause of mortality and morbidity in these victims. According to recent reports in Iran, $73 \%$ of mortality in burn patients are due to infections. ${ }^{15}$ In spite of acceptable management of infection control strategies in burn patients, colonization and proliferation of microorganisms still threaten the patient's life. One of the important causes of microbial invasion and failure of infection control policies is due to bacterial resistance to antimicrobial agents. With regards to the importance of nosocomial infections as the main threat for burn patients, diagnosing the pattern of prevalent microorganisms with their resistance algorithm in hospital settings may improve therapeutic decisions. ${ }^{16,17}$ According to the fact that a broad spectrum of microorganisms can cause infection in burns, many literature studies declare that the microbiological profile of the organisms changes over the time of hospitalization in burn patients. In new hospitalized burn cases, Gram-positive organisms are more predominant at first. But in prolonged hospitalization, Gram-negative bacteria become more prevalent. ${ }^{18}$ Based on different documents it has been shown that Gram-positive infections are from endogenous sources and this is while, with prolonged hospitalization and broad-spectrum antibiotic use, resistant nosocomial infections from exogenous sources are replaced. Our recent findings revealed that the most common nosocomial pathogens in Shiraz burn center are as follows: P. aeruginosa, Klebsiellasp., A. baumannii and S. aureus. Infection in these patients is not only the main reason for mortality but also for extending the length of hospitalization stay. Available evidence asserts that a longer stay is associated with more infections; also, the survival rate for uninfected patients is two times more than for infected victims. ${ }^{19}$

Comparing these results with other near board countries has shown similar bacterial species patterns. Moreover, many studies identified $P$. aeruginos $a$ isolates as the most common pathogen in burn injuries. ${ }^{20-22}$ In contrast, A. baumannii has been reported to prevail in Asian and Arab countries. In some other reports, S. aureus was defined as the leader of nosocomial infection in burn cases. ${ }^{23}$

In comparing the current results with a previous study in southwest Iran, $P$. aeruginosa is still in similar rank and this is while Klebsiellasp. has been shifted to the second level of infections. Although the pattern of infection in the mentioned center is not changed approximately, some points are highlighted: the numbers of P. aeruginosa, Klebsiellasp. and $S$. aureus isolates have increased, and this is while some 
infections such as $A$. baumannii have gradually waned during the study period. ${ }^{18,24}$

Evaluating the antibiogram profile of infections, in comparison with a previous report, has shown a considerable achievement in decreasing the resistance pattern in prevalent infectious agents. ${ }^{18}$ Although there are an increasing number of some infections, for instance $P$. aeruginosa and Klebsiella sp. significantly, this is similar with other burn centers around the world in recent years ${ }^{20,21}$ Due to the aim of the study, it was revealed that this achievement has been gained due to a strict politicized infection control and stewardship program in the appointed burn center. In this policy we managed to stop the empirical antibiotic treatment until the third day of hospitalization and decreased the use of irrational antibiotics based on prevalent infections in our center. In some conditions we utilize clean care of wounds and use of antibacterial dressing instead of systematic antibiotic therapy, which helps to latent the antibiotic use time for longer.

The emergence of antimicrobial resistance has become one of the major challenges and global concerns. Antibiotic resistance in nosocomial infections has been associated with prolonged suffering, economic and social burdens, and significantly higher mortality. ${ }^{25}$ Switching the prescribed drug without microbial culture, use of prophylactic antibiotics, and excessive and inappropriate use of broad-spectrum antibiotics are the main reasons for antibiotic resistance in clinical settings. Antimicrobial stewardship programs along with infection control protocols (ie hand hygiene, keeping infected patients in isolate rooms, wound clean care and local antibiotic use) could help to control infections and restrict resistant infection outbreaks in burn centers. ${ }^{26}$

Although many infection control and stewardship policies are accountable to decrease the prevalent infections and resistance agents, it is of considerable importance to also be vigilant with patients who are transferred from other centers, which can break the infection control chain. For this group of patients, it has been recommended that they are kept in private and isolate rooms until the results of their culture are known and first treatments have been performed for decreasing bacterial colonizations and infections. In pediatric wards, more restricted policies must be considered for mobile gadgets like toys. ${ }^{27}$

According to the conclusion of the current results, focusing on antibiotic treatment of patients especially in prophylaxis form or irrational empirical treatment will raise resistance to nosocomial infection and its control will be more difficult. It is very important to know that antibiotic resistance is accelerated by the misuse and overuse of antibiotics, as well as poor infection prevention and control. Based on this, we must try to perform clean care treatments and do strict infection control programs especially in burn settings, with the following comments: $:^{28}$

1) design an action plan to tackle antibiotic resistance; 2) improve surveillance of antibiotic-resistant infections; 3) strengthen policies, programs and implementation of infection prevention and control measures; 4) regulate and promote the appropriate use and disposal of quality medicines; and 5) make information available on the impact of antibiotic resistance for clinicians who prescribe antibioti

\section{Conclusion}

Appropriate infection control policies, especially clean care, could be helpful for decreasing the infection rate and resistance pattern and to optimize patient care. Moreover, knowing the antimicrobial pattern of resistance in burn centers can provide suitable treatment for these victims. Knowing the resistance pattern in the hospital setting, we can manage the use of antibiotics in kind, time and dose.

\section{Funding}

The authors received no financial support for the research, authorship, or publication of this article.

\section{Disclosure}

The authors declare that they have no conflicts of interest in this work.

\section{References}

1. Leseva M, Arguirova M, Nashev D, Zamfirova E, Hadzhyiski O. Nosocomial infections in burn patients: etiology, antimicrobial resistance, means to control. Ann Burns Fire Disasters. 2013;26(1):5.

2. Church D, Elsayed S, Reid O, et al. Burn wound infections. Clin Microbiol Rev. 2006;19(2):403-434. doi:10.1128/CMR.19.2.403434.2006

3. Lachiewicz AM, Hauck CG, Weber DJ, et al. Bacterial infections after burn injuries: impact of multidrug resistance. Clin Infect Dis. 2017;65 (12):2130-2136. doi:10.1093/cid/cix682

4. Pednekar A, Paul M. K, Prakash JAJ, et al. Emerging trends of antimicrobial susceptibility and resistance in burn patients. Burns Open. 2019;3(2):51-55. doi:10.1016/j.burnso.2019.01.002

5. Pruitt Jr BA. The diagnosis and treatment of infection in the burn patient. Burns. 1984;11(2):79-91. doi:10.1016/0305-4179(84)90 129-3

6. Russotto V, Cortegiani A, Fasciana T, et al. What healthcare workers should know about environmental bacterial contamination in the intensive care unit. Biomed Res Int. 2017;2017.

7. Fasciana T, Gentile B, Aquilina M, et al. Co-existence of virulence factors and antibiotic resistance in new Klebsiella pneumoniae clones emerging in south of Italy. BMC Infect Dis. 2019;19(1):928. doi:10. 1186/s12879-019-4565-3 
8. Carmeli Y. Strategies for managing today's infections. Clin Microbiol Infect. 2008;14:22-31. doi:10.1111/j.1469-0691.2008.01957.x

9. Zhang L, Kinkelaar D, Huang Y, et al. Acquired antibiotic resistance: are we born with it? Appl Environ Microbiol. 2011;77(20):7134-7141. doi:10.1128/AEM.05087-11

10. Zaha DC, Kiss R, Hegedüs C, et al. Recent advances in investigation, prevention, and management of healthcare-associated infections (HAIs): resistant multidrug strain colonization and its risk factors in an intensive care unit of a University hospital. Biomed Res Int. 2019;2019.

11. Wanis M, Walker SAN, Daneman N, et al. Impact of hospital length of stay on the distribution of Gram negative bacteria and likelihood of isolating a resistant organism in a Canadian burn center. Burns. 2016;42(1):104-111. doi:10.1016/j.burns.2015.07.010

12. Alaghehbandan R, Azimi L, Lari AR. Nosocomial infections among burn patients in Teheran, Iran: a decade later. Ann Burns Fire Disasters. 2012;25(1):3.

13. Norbury W, Herndon DN, Tanksley J, et al. Infection in burns. Surg Infect (Larchmt). 2016;17(2):250-255. doi:10.1089/sur.2013.134

14. Clinical and Laboratory Standards Institute. Performance Standards for Antimicrobial Susceptibility Testing, in Test for Methicillin Resistance (Oxacillin Resistance) in Staphylococcus Spp. Wayne, PA 19087 USA: CLSI; 2018:126-133.

15. Sabzghabaee AM, Karamyafti M, Abedi D, et al. Antimicrobial resistance pattern of bacterial isolates from burn wounds in an Iranian University Hospital. J Res Pharm Pract. 2012;1(1):30. doi:10.4103/2279-042X.99675

16. Weber J, McManus A; Nursing Committee of the International Society for Burn Injuries. Infection control in burn patients. Burns. 2004;30(8):A16-A24. doi:10.1016/j.burns.2004.08.003

17. Weinstein RA, Mayhall CG. The epidemiology of burn wound infections: then and now. Clin Infect Dis. 2003;37(4):543-550. doi:10.1086/376993

18. Pirbonyeh N, Bazargani A, Emami A, et al. Cross sectional study of burn infections and antibiotic susceptibility pattern for the improvement of treatment policy. J Patient Saf Qual Improv. 2017;5 (2):535-541.
19. Alp E, Coruh A, Gunay GK, et al. Risk factors for nosocomial infection and mortality in burn patients: 10 years of experience at a university hospital. J Burn Care Res. 2012;33(3):379-385. doi:10.1097/BCR.0b013e318234966c

20. Chaudhary NA, Munawar MD, Khan MT, Rehan K, Sadiq A. Epidemiology, bacteriological profile, and antibiotic sensitivity pattern of burn wounds in the burn unit of a tertiary care hospital. Cureus. 2019;11(6).

21. Oncul O, Ulkur E, Acar A, et al. Prospective analysis of nosocomial infections in a burn care unit, Turkey. Indian J Med Res. 2009;130 (6):758-764.

22. Kerr KG, Snelling AM. Pseudomonas aeruginosa: a formidable and ever-present adversary. $J$ Hosp Infect. 2009;73(4):338-344. doi:10.1016/j.jhin.2009.04.020

23. Guggenheim M, Zbinden R, Handschin AE, Gohritz A, Altintas MA, Giovanoli P. Changes in bacterial isolates from burn wounds and their antibiograms: a 20-year study (1986-2005). Burns. 2009;35 (4):553-560. doi:10.1016/j.burns.2008.09.004

24. Pirbonyeh N, Emami A, Bazargani A, Javanmardi F, Hosseini SM, Derakhshan B. Integron related resistance in new emerged Staphylococcus lugdunensis infection in burn patients. J Burn Care Res. 2019

25. Javanmardi F, Emami A, Pirbonyeh N, et al. Study of multidrug resistance in prevalent Gram-negative bacteria in burn patients in Iran: a systematic review and meta-analysis. $J$ Glob Antimicrob Resist. 2019;19:64-72. doi:10.1016/j.jgar.2019.04.017

26. Rafla K, Tredget EE. Infection control in the burn unit. Burns. 2011;37(1):5-15. doi:10.1016/j.burns.2009.06.198

27. Ahuja RB, Gibran N, Greenhalgh D, et al. ISBI practice guidelines for burn care. Burns. 2016;42(5):953-1021. doi:10.1016/j.burns.2016. 05.013 .

28. Rowan MP, Cancio LC, Elster EA, et al. Burn wound healing and treatment: review and advancements. Crit Care. 2015;19(1):243. doi:10.1186/s13054-015-0961-2
Infection and Drug Resistance

\section{Publish your work in this journal}

Infection and Drug Resistance is an international, peer-reviewed openaccess journal that focuses on the optimal treatment of infection (bacterial, fungal and viral) and the development and institution of preventive strategies to minimize the development and spread of resistance. The journal is specifically concerned with the epidemiology of

\section{Dovepress}

antibiotic resistance and the mechanisms of resistance development and diffusion in both hospitals and the community. The manuscript management system is completely online and includes a very quick and fair peerreview system, which is all easy to use. Visit http://www.dovepress.com/ testimonials.php to read real quotes from published authors. 